\title{
RESEARCH OF PHYSIOLOGICAL FACTORS AFFECTING PILOT PERFORMANCE IN FLIGHT SIMULATION TRAINING DEVICE
}

\begin{abstract}
Physiological factors can easily affect a pilot performance and jeopardise the safety of the flight. Therefore, the research is based on the observation of student pilots' decision making and flying abilities during different flight conditions predetermined for the flight in simulation training device. The use of flight simulation training device is helpful as it is possible to vary flight parameters and consequently observe the pilot's reactions to different situations. The combination of pilot error statistics and subjective questionnaire enable to determine the influence of external conditions on the pilot's psychological state and subsequent performance during critical flight tasks.
\end{abstract}

Keywords: Training, fatigue, pilot performance, simulation training device.

\section{Introduction}

Many health factors and physiological effects are linked with flying. Some of them are minor, whilst others require a focus of attention in order to ensure safety of the flight. Moreover, there are a large variety of factors that may cause unsafe behaviour that will cause unsafe flight operations. Most of the worst pilot performances that still occur are attributed to human errors and therefore it is inevitable to analyse human error, workload, stress and other physiological conditions and their relationship with pilot performance during flight tasks [1]. Generally, the human being is most reliable under adequate levels of workload that do not change suddenly, unpredictable and immediately when the workload is excessive [2]. Errors arise from the inability of the human operators to deal with high information rates that come from the external environment [3].

\subsection{Influence of physiological factors on pilot performance}

For the purpose of this research, we focus on the performance measurements monitoring pilot behaviour during changing predetermined physiological conditions in order to seek and determine the level of successful or unsuccessful flight tasks.

Moreover, workload for a pilot varies due to the diverse features of the flight - different phases of flight represent different workloads for the pilot. For instance, Instrument Flight Rule (IFR) flight creates significantly higher workload than Visual
Flight Rule (VFR) flight. Also, pilots show higher workload for the Take-off phase or Landing phase compared to Cruise or VFR flight phase. Indeed, workload is modified further through other factors that are not under the control of the flight crew, such as weather conditions, visibility, traffic density or even communication requirements [4]. However, physiological factors may cause change and degradation of basic cognitive abilities and it can also degrade the pilot's rational thinking and concentration that lead towards higher error rate during the flight [5]. On the other hand, it also requires the ability of the pilot to depersonalise himself from the adverse effects of critical situations and keep a cool mind, clear and correct evaluation of current flight situations. The influence of those factors prolongs the reaction time that is linked with later pilot performance of checklist and it also increases the number of pilot errors [6].

This paragraph deals with measured physiological and psychological factors, such as heat, noise, hypoglycaemia and fatigue. These factors were chosen due to the fact that it is easy to activate them with the FSTD environment in simulation training devices. During the 24 hours prior to blood sugar measurement, the pilots had a limited consumption of sugar and they also started without food-intake. Moreover, blood sugar was measured, where specific values of all pilots are illustrated in the following subchapters and the impact of this factor on the ability and flight control accuracy during the final landing phase is detailed. The impact of noise was simulated by enhanced engine sounds and by ongoing communication (radio chatter) from the ground air traffic control. Pilot workload was increased by the need to confirm the dispatcher's instructions. Moreover, any phase of

\footnotetext{
* Andrej Novak, Maria Mrazova

Air Transport Department, University of Zilina, Slovakia

E-mail: andrej.novak@fpedas.uniza.sk
} 
flight is accompanied by noise, especially during take-off phase and also during the landing, by use of the thrust reversers. Fatigue measurement was performed after high physical and mental pilot workload (was simulated after at least 24 hours conditions based on continuous wakefulness) which caused the individual's lower concentration during flight tasks and it also delayed responses during simulated crisis situations. In the case of heat measurements and their impact on pilot performance we used environmental conditions based on increased air temperature inside the FSTD cockpit temperatures of circa $47^{\circ} \mathrm{C}$ with the aid of an external $9 \mathrm{~kW}$ heat exchanger normally used for heating the air of the engine during the winter season.

\section{Research methodology}

\section{Flown trajectory}

For the purpose of pilot performance measurements using an FSTD $f$ we chose ILS approach procedure at the airport Zilina - Dolny Hricov (LZZI) for runway 06 as the base procedure during which the measurement will take place. This procedure was chosen for several reasons. Firstly, in this procedure there are segments with prescribed flight altitude and final approach segment with a clearly defined glide path angle allowing measurable deviations from both the glide slope and localizer. This will allow well-defined, measurable parameters for evaluation of performance. Secondly, majority of the pilots surveyed in this activity are familiar with this procedure, as they are actively flying this approach procedure during their training at the Air Training and Education Centre of the University of Zilina and often perform these approaches during their active career. Therefore, this procedure eliminated varying efficiency due to unfamiliarity with the approach procedure i.e. any learning effect. Lastly, the procedure is not monotonous, and by its nature every precision approach procedure produces significant load on the pilot.

Every consequential approach was followed by missed approach procedure, as published in the approach chart. It eliminated the documentation required for the flight to a single chart, linking the approach procedure with the missed approach.

\subsection{Procedure for objective measurement}

\section{Measured variables}

For the purpose of objective measurement without being influenced by changes in other external factors, it was necessary to find appropriate variables that are only affected by the measured physiological influence and the resulting pilot state.

Three variables were created and they were based on the number of pilot errors in the flown segment. The first variable is measured at relatively lower workload. It is the number of exceedance of the assigned flight altitude by more than 100 feet $^{1}$ (ft) in the outbound segment of initial approach after crossing the NDB - non-directional radio beacon ZLA to the final approach point. The measurements were carried out in 10-second intervals in order to also capture the deviation length.

The second variable measured at lower workload is the number of speed exceedance greater than 10 knots. The assigned speed for this segment is 200 knots and measurements were carried out at 10-second intervals. The third measurement was carried out in the time of high workload during the approach for landing. The measured variable is the deviation from the glide path $(\mathrm{G} / \mathrm{S})$ by more than one dot on the indicator. This deviation was monitored at intervals of $100 \mathrm{ft}$ in height, and from $3900 \mathrm{ft}$ to $1700 \mathrm{ft}$. Similarly, the fourth variable was measured simultaneously in the same phase, it was characterised as a departure from the lateral guidance (LOC) by more than one dot on the indicator. This deviation was monitored at intervals of $100 \mathrm{ft}$ in height, and from $3900 \mathrm{ft}$ to $1700 \mathrm{ft}$. The last measured variable was deviation from the reference airspeed of more than 10 knots, where the assigned airspeed for the purpose of measuring was set to 140 knots. This deviation was monitored at intervals of $100 \mathrm{ft}$ in height, and from $3900 \mathrm{ft}$ to $1700 \mathrm{ft}$.

\section{Processing of measurement}

The measurement was processed by statistical methods. We used the mean that was obtained by dividing the sum of observed values by the number of observations. For each pilot (pilot 1, 2, $3,4,5,6$ and 7) we count the mean for each factor (reference ${ }^{2}$, hypoglycaemia, fatigue, heat and noise). Consequently, we count the average for all the data that was obtained for a particular influence.

\section{Research outcomes}

As mentioned above, the subjects of our research were seven pilots with different amount of flying hours. The flight was evaluated in 2 phases - initial and intermediate approach segment and also final approach segment. An instructor was monitoring the student pilot's behaviour during selected flight phases where external factors were changed after every 5 final approaches. For each physiological factor we count the average values that provide us with factors that have the most severe impact on the pilot performance during the flight tasks (Table 1).

${ }^{1}$ Feet $(\mathrm{ft})$ - a unit of length. It makes 0.3048 meters.

${ }^{2}$ Reference - for the purpose of this research, reference means the flight without any external conditions that could have any impact on pilot performance. 
Illustration of the total average values related to external researched factors [author]

Table 1

\begin{tabular}{|l|c|c|c|c|}
\hline Influence & Altitude & Airspeed & ILS & Total average \\
\hline Reference & 1.90 & 1.36 & 2.10 & 5.36 \\
\hline Noise & 1.94 & 1.82 & 2.97 & 6.74 \\
\hline Hypoglycaemia & 3.06 & 3.00 & 3.97 & 10.03 \\
\hline Heat & 2.03 & 2.29 & 6.69 & 11.00 \\
\hline Fatigue & 2.17 & 1.17 & 8.69 & 12.03 \\
\hline
\end{tabular}

Reference column represents average number of pilot errors during the whole measurement cycle without any aggravated conditions for the selected flight task. Consequently, other columns (Noise, Hypoglycaemia, Heat and Fatigue) represent an effect of changing aggravated conditions that has different impact on the pilot performance during the intermediate and final approach. Following Fig. 1 we can see that the highest impact of fatigue factor that was mostly visible during ILS approach linked with deviations from the flight altitude by more than 100 feet and it was also followed by deviations from the specified airspeed - by more than 10 knots.

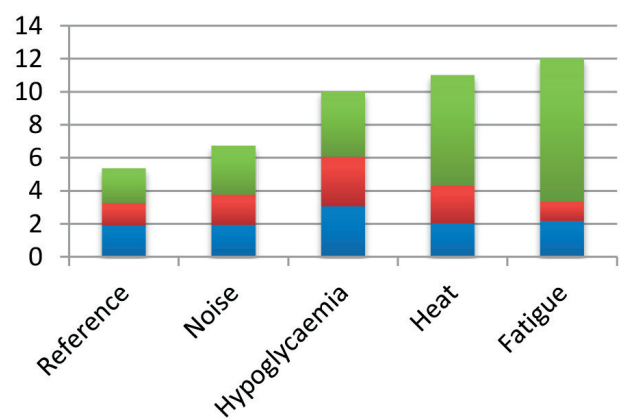

ILS

Airspeed

altitude

Fig. 1 Illustration of researched physiological factors [author]

\section{NOTE:}

All figures have the same graphical structure that is based on these characteristics: the $\mathrm{x}$-axis represents researched physiological factors (reference, noise, hypoglycaemia, heat and fatigue), whereas the y-axis shows number of pilot errors.

In addition, Figs. 2, 3 and 4 represent three researched parameters that were regarded (altitude, airspeed and ILS). As can be seen in Fig. 2, according to Altitude parameter it is obvious that many pilot's errors were made due to the hypoglycaemia effect. Figure 3 illustrates that Airspeed parameter shows the same hypoglycaemia effect. According to Fig. 4 we can see that ILS parameter (sum of glide path errors, localizer errors and air speed errors) in the final approach segment was mostly influenced by the fatigue factor. Today, the issue of fatigue is still being argued and therefore this research also concentrates on this parameter as one of the serious problems in an effort to decrease the risks associated with it.

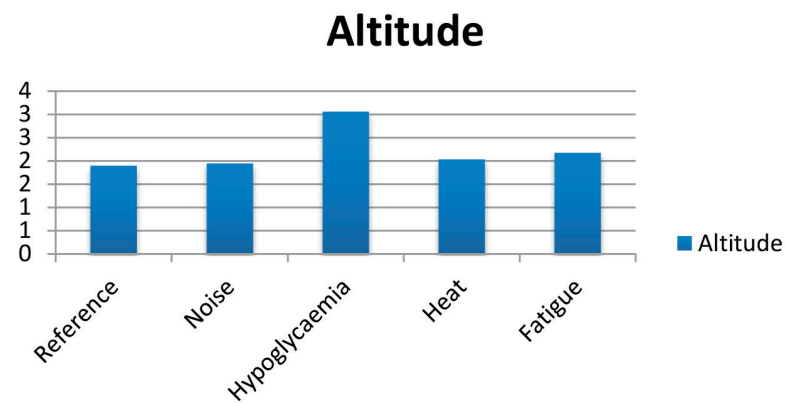

Fig. 2 Impact of physiological factor on Altitude [author]

\section{Airspeed}

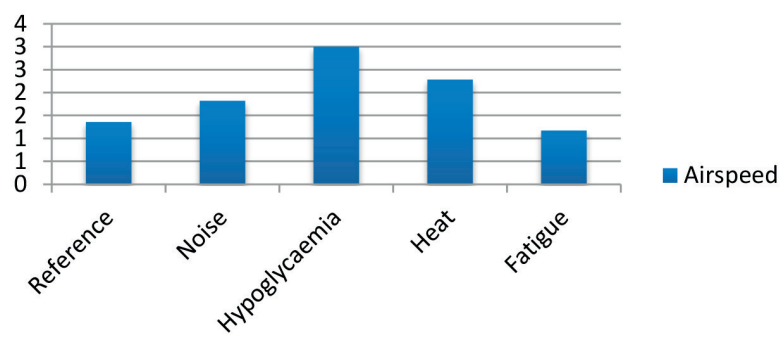

Fig. 3 Impact of physiological factor on Airspeed [author]

\section{ILS}

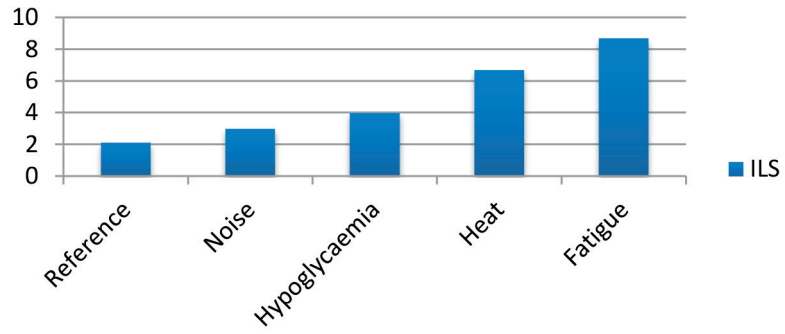

Fig. 4 Impact of physiological factor on ILS [author]

Moreover, Pilot 1 has about 300 flying hours and as can be seen in Fig. 5, the most significant factor that influenced Pilot 1 during the flight tasks was fatigue mainly during the final approach segment (ILS parameter). The Pilot's errors related to Airspeed parameter were influenced mostly by the noise factor and Altitude parameter was affected by the fatigue factor.

Furthermore, Pilot 2 has about 50 flying hours and his flight tasks was influenced during the final approach segment, similarly to the case of Pilot 1, by the fatigue factor. Consequently, Altitude parameter was affected in the same way by hypoglycaemia and noise factors. Equally, Airspeed parameter was influenced by the noise factor as in the case of Pilot 1 . 
Pilot 1

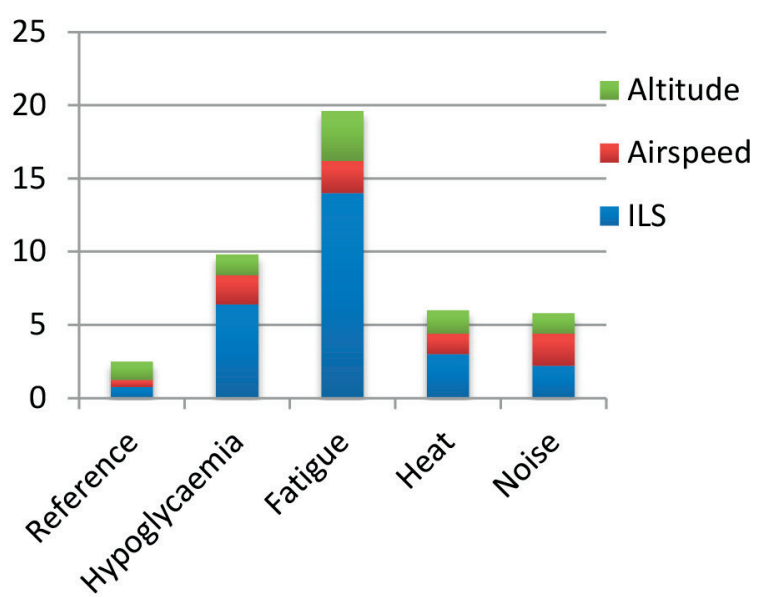

Pilot 2

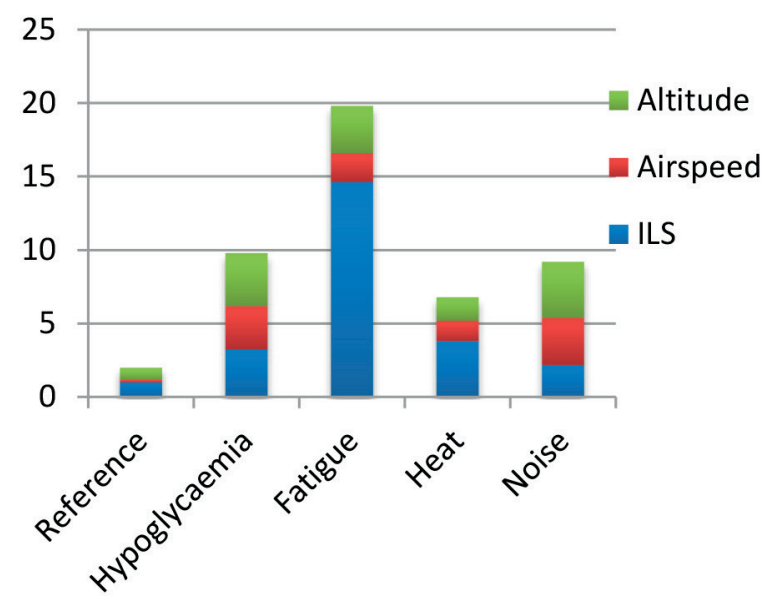

Fig. 5 Illustration of physiological factors' impact on performance related to Pilot 1 and Pilot 2 [author]

Pilot 3

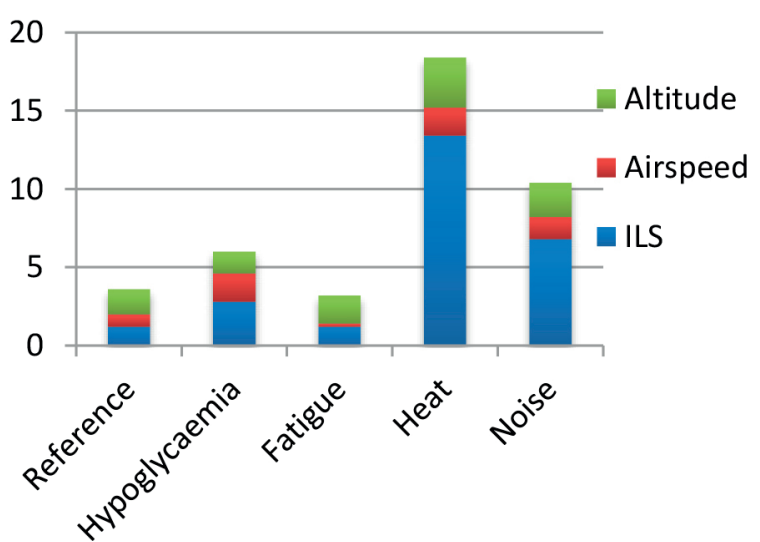

Pilot 4

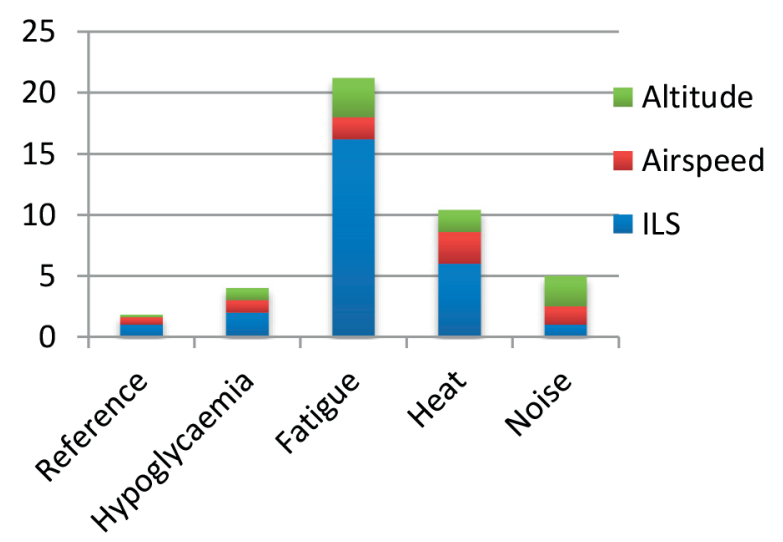

Fig. 6 Illustration of physiological factors' impact on performance related to Pilot 3 and Pilot 4 [author]

Pilot 5

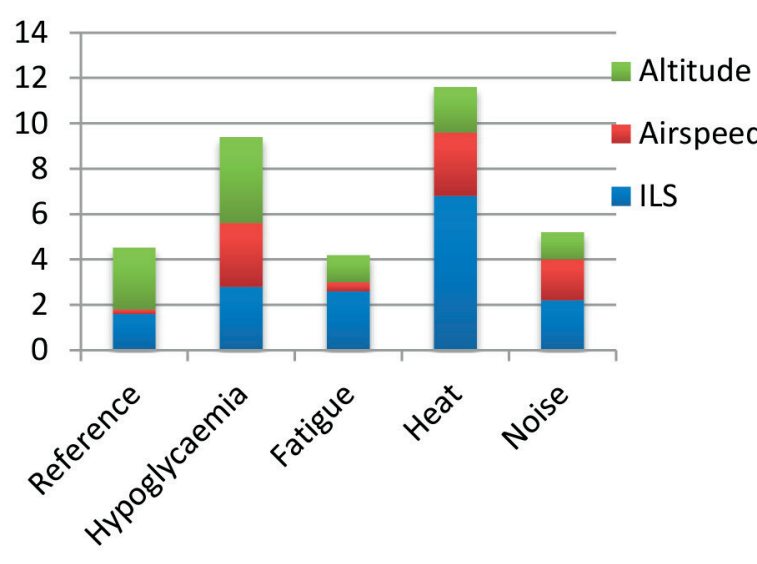

Pilot 6

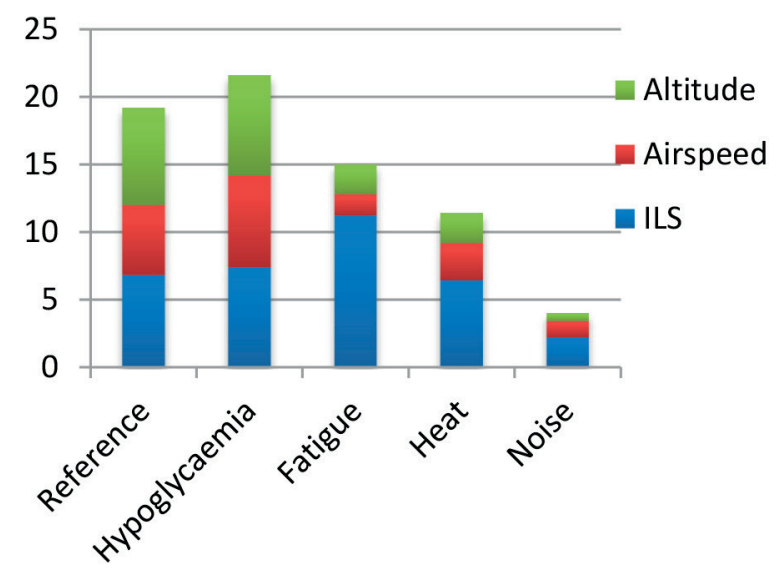

Fig. 7 Illustration of physiological factors' impact on performance related to Pilot 5 and Pilot 6 [author] 
Figure 6 illustrates the performance of Pilot 3 and Pilot 4. In the case of Pilot 3 (about 150 flying hours) it is evident that the most significant impact was caused by the heat represented by ILS and Altitude parameter, while Airspeed parameter was mostly influenced by the hypoglycaemia factor. Pilot 4 has about 160 flying hours and his performance was mostly influenced by the fatigue factor during the final approach segment. Also, the pilot has many errors due to the heat factor (when Airspeed parameter was researched) and Altitude parameter was influenced mostly by the noise and fatigue factors.

\section{Pilot 7}

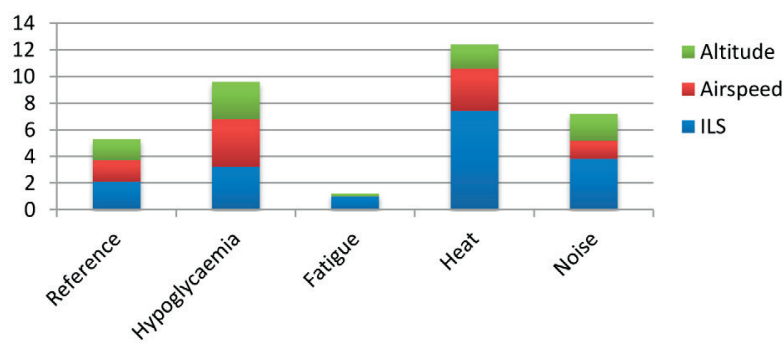

Fig. 8 Illustration of physiological factors' impact on performance related to Pilot 7 [author]

Figure 7 illustrates the performance of Pilot 5 and Pilot 6 . Firstly, Pilot 5 has about 160 flying hours and ILS parameter was mostly influenced by heat factor while altitude parameter was mostly affected by hypoglycaemia effect. Besides, Pilot 6 has about 155 flying hours and in this case ILS parameter was mostly influenced by fatigue factor while altitude parameter was affected by the hypoglycaemia factor. Pilot 7 has about 45 flying hours and his performance was mostly influenced by heat factor whereas Altitude and Airspeed parameters were affected by the hypoglycaemia factor, as can be seen in Fig. 8 above.

\section{Conclusion}

Firstly, this research has shown that the most significant impacts were caused by the fatigue and hypoglycaemia factors. The other selected factors had just minor impact on the pilots' performance. We can also see that pilots' errors were most notable during the final approach segment where the ILS parameters were studied. Moreover, all measurements and their related features were measured during 6 months in an attempt to characterise different individual mental states of the tested student pilots and also in order to estimate the activity of the central nervous system (CNS) related to the defined flight tasks.

Secondly, the pilot's performance decrements resulted more from inadequate interface than from a depletion of mental resource. Moreover, it is necessary to realise that the pilot has to hold a large amount of important information in his working memory while attending to another task, such as answering a radio call and this is indicative of a mental workload problem. During our research it often happened due to the fact that pilots were required to do a flight task that was not expected. As the pilots were selected according to different amounts of flying hours, our research does not confirm that the number of pilots' errors is proportional to the number of flying hours, but it unfolds from the physical and mental conditions of the pilot.

In brief, practical contribution of this research offers another possibility to extend the group of studied physiological and psychological factors, such as heart rate, mental fatigue or drowsiness in order to improve the safety of flight operations.

\section{Acknowledgement}

This paper is published as one of the scientific outputs of the project: "The research on virtual reality elements application: the significant improvement of simulator performance characteristics", ITMS: 26220220167“.

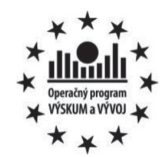

\section{References}

[1] ENDSLEY, M.: Measurement of Situational Awareness in Dynamic Systems. Human Factors 37 (1), p. 33, 1995.

[2] GANDER, P. H., NGUYEN, D., ROSEKIND, M. R., CONNELL, L.j: Age, Circadian Rhythms, and Sleep Loss in Flight Crews. Aviation, Space and Environmental Medicine 64 (3), pp. 189-190, 1993.

[3] YEH, Y. Y., WICKENS, CH. D.: Dissociation of Performance and Subjective Measures of Workload and Performance. Human Factors 30, pp. 678-679, 1988.

[4] WILSON, G. F.: Air-to-ground Training Missions: A Psychophysiological Workload Analysis. Ergonomics 36 (9), pp. 1072-1073, 1993.

[5] BENO, L., BUGAJ, M., NOVAK, A.: Application of RCM Principles in the Air Operations, Communications - Scientific Letters of the University of Zilina, vol. 7 (2), 24 p., 2005.

[6] BORGHINI, G., ASTOLFI, L., VECCHIATO, G., MATTIA, D.: Measuring Neurophysiological Signals in Aircraft Pilots for the Assessment of Mental Workload, Fatigue and Drowsiness. Neuroscience and Biobehavioral Reviews, pp. 3-5, 2012. 